SLAC-PUB-8129

April 1999

\title{
Multi-Bunch Instability Diagnostics via Digital Feedback Systems at PEP-II, DA $\Phi$ NE, ALS and SPEAR *
}

\author{
J. Fox $^{\dagger}$, R. Larsen, S. Prabhakar, D. Teytelman, A. Young \\ Stanford Linear Accelerator Center, Stanford University, Stanford, CA 94309 \\ A. Drago, M. Serio \\ INFN Laboratori Nazionali di Frascati, 00044 Frascati (Roma), Italy \\ W. Barry, G. Stover \\ LBL, 1 Cyclotron Road, Berkeley, CA 94563
}

\begin{abstract}
Longitudinal feedback systems based on a common programmable DSP architecture have been commissioned at 4 laboratories. In addition to longitudinal feedback and beam diagnostics these flexible systems have been programmed to provide diagnostics for tranverse motion. The diagnostic functions are based on transient domain techniques which record the response of every bunch while the feedback system manipulates the beam. Operational experience from 4 installations is illustrated via experimental results from PEP-II, DA $\Phi$ NE, ALS and SPEAR. Modal growth and damping rates for transverse and longitudinal planes are measured via short $(20 \mathrm{~ms})$ transient excitations for unstable and stable coupled-bunch modes. Data from steady-state measurements are used to identify unstable modes, noise-driven beam motion, and noise sources. Techniques are illustrated which allow the prediction of instability thresholds from low-current measurements of stable beams. Tranverse bunch train grow-damp sequences which measure the time evolution of instabilities along the bunch train are presented and compared to signatures expected from ion and fast ion instabilities.
\end{abstract}

Invited talk presented at the IEEE Particle Accelerator Conference (PAC99)

New York, New York

March 29 - April 2, 1999

*Work supported by Department of Energy contract DE-AC03-76SF00515. 


\section{Transient-Domain Diagnostics}

High current colliders such as the B Factories and $\Phi$-factory, and high current light sources such as the ALS have pushed accelerator operation into regimes where coupled-bunch instabilities are operational concerns [1,2]. Several of these facilities use active feedback to control longitudinal and transverse instabilities [3, 4]. Diagnostic techniques to understand thresholds for instability and diagnostics to understand the performance of the feedback systems have become important tools for operation of these facilities.

The longitudinal feedback systems developed for the PEP-II/DA $\Phi$ NE/ALS machines use a digital processing architecture. This programmable processing block allows the recording of beam motion in conjunction with feedback or special diagnostic algorithms [5]. The information present in the oscillation co-ordinates is processed off-line to quantify the motion of the bunches. Information may be found in the steady-state controlled motion (information such as residual noise) or from dynamic sequences where the beam might be excited to some amplitude, then damped back to the controlled state.

Our group has recently applied these transient-domain diagnostics to transverse instability measurements. We have used the existing ALS and PEP-II transverse feedback systems to control the beams in conjunction with the longitudinal processing hardware acting as a high-speed data recorder. In this scheme the digital processing block does not compute correction signals, though the digital hardware does control various gating functions in the transverse processing to synchronise the recording of excited/damped bunch motion. [6]

The longitudinal system design is based on downsampled processing, in which the sampling rate of the digital processing is matched to the oscillation frequency of the bunches, rather than the revolution frequency. Downsampled processing is advantageous for the longitudinal systems where the synchrotron oscillation frequency is significantly lower than the revolution frequency. The transverse systems operate as aliased sampling systems, as the revolution (sampling) frequency is lower than the natural betatron oscillation frequencies. The recording of this aliased information in the downsampled data recorder further aliases and folds the spectral information. However, if the machine tune is a known quantity and the downsampling factor is selected appropriately, downsampled recording loses no useful spectral information. The off-line data processing can completely recover the original oscillation frequencies and phase relationships in the bunches. Another way of thinking of the bandwidth required in the transverse motion recording process is that one must sample fast enough to unambigously record the envelope of any signal modulation corresponding to bunch motion, though the carrier frequency of the bunch motion may not be recorded. The envelopes contain all the spectral information from which growth/damping rates may be calculated. Additionally, the sampling process correctly preserves the phase information in the bunch oscillations, so that the true modal information relating the phase relationships of the bunches is faithfully preserved in the analysis. 


\section{a) Osc. Envelopes in Time Domain}

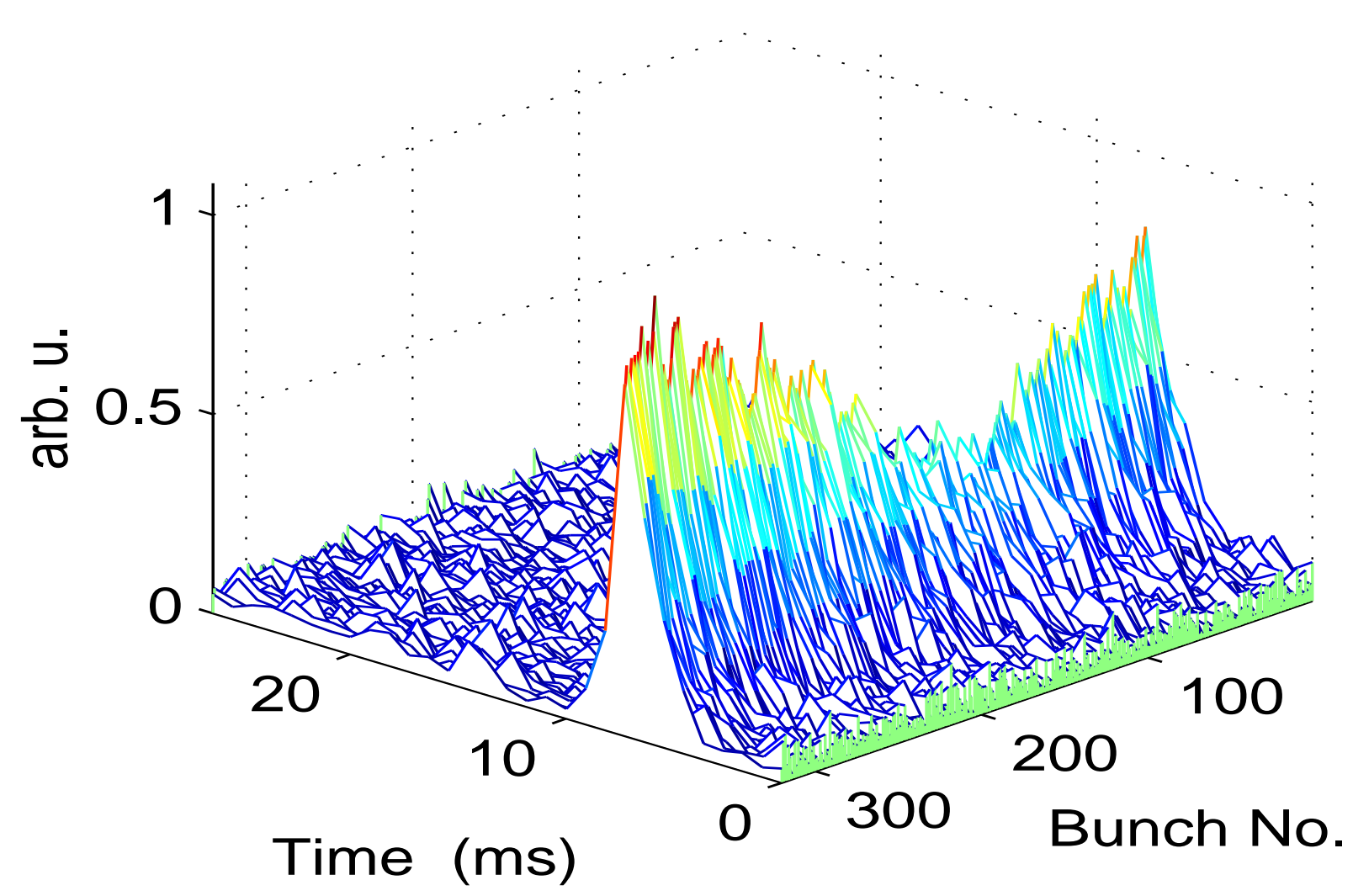

Figure 1: . Grow-Damp sequence in the horizontal plane from the ALS. The envelope of the bunch motion shows the free growth $(0<t<6 \mathrm{~ms})$, then damping under the action of feedback $(t>6 \mathrm{~ms})$

\section{Modal growth and damping rates for transverse and longitudinal planes}

The most direct measurement of unstable motion is found from grow-damp sequences, in which a controlled beam is allowed to grow in amplitude without feedback for a short interval, followed by a damping transient as feedback control is re-applied. Fourier-transform techniques allow the identification of particular unstable modes of motion from the bunch data representation [7]. An example from the the ALS reveals the growth of two transverse unstable modes in figures \#1 and \#2. The resistive-wall impedance is the dominant mechanism driving this instability, as revealed in the strongest growth of modes 326 and 327 . The damping transient in this figure directly reveals the available gain margin in the feedback channel, as the damping rates are seen to be roughly $10 \mathrm{X}$ the growth rates for this current. Figure \#3 shows a longitudinal damp-grow transient from the DA $\Phi$ NE machine, which shows a system controlled by feedback from which unstable mode 20 motion rapidly grows as the feedback is turned off. 


\section{b) Evolution of Modes}

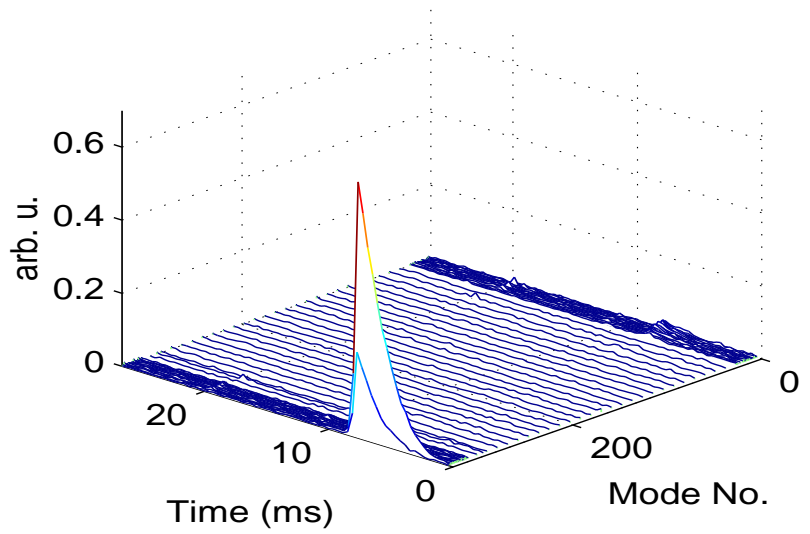

Figure 2: . The recorded bunch motion is Fourier transformed to reveal the growth of modes 326 and 327.

One important feature of these transient-domain techniques is that the full spectrum of bunch motion is recorded in a single transient, unlike a narrowband measurement made with a traditional spectrum analyser. Another important feature of this technique is that motion can be studied in the small-oscillation (linear) situation before saturation and non-linear mechanisms appear.

\section{Noise-driven beam motion}

Useful information can also be extracted from steady-state records of controlled motion. The controlled system will show the signature of any disturbances driving the beam, attenuated by the action of the feedback system. In the longitudinal plane, motion of the beam might be due to true unstable motion from HOMs, or motion might be due to noise injected from the RF system. Our experience from the PEP-II LER commissioning illustrates these issues. Figure \#4 shows a plot of LER modal amplitude (feedback on) vs. time for a 291 bunch even fill. There is significant structure in the data, suggesting that whatever mechanism is driving the beam at mode zero is a noisy or non-stationary process. Further experiments with the RF system revealed a $10 \mathrm{~Hz}$ periodic phase modulation of the master oscillator, which was first identified from the beam spectral information. After improvement of the master oscillator phase noise [8] and implementation of a low-mode "woofer" through the RF system [9], the resulting all-mode rms noise floor is seen in figure \#5. With these improvements the residual noise level for mode zero is at the 0.03 degree $\mathrm{rms}$ ( at $476 \mathrm{MHz}$ ) level, while higher modes fall to 0.005 degrees rms which is the electronic noise floor of the processing channel. 


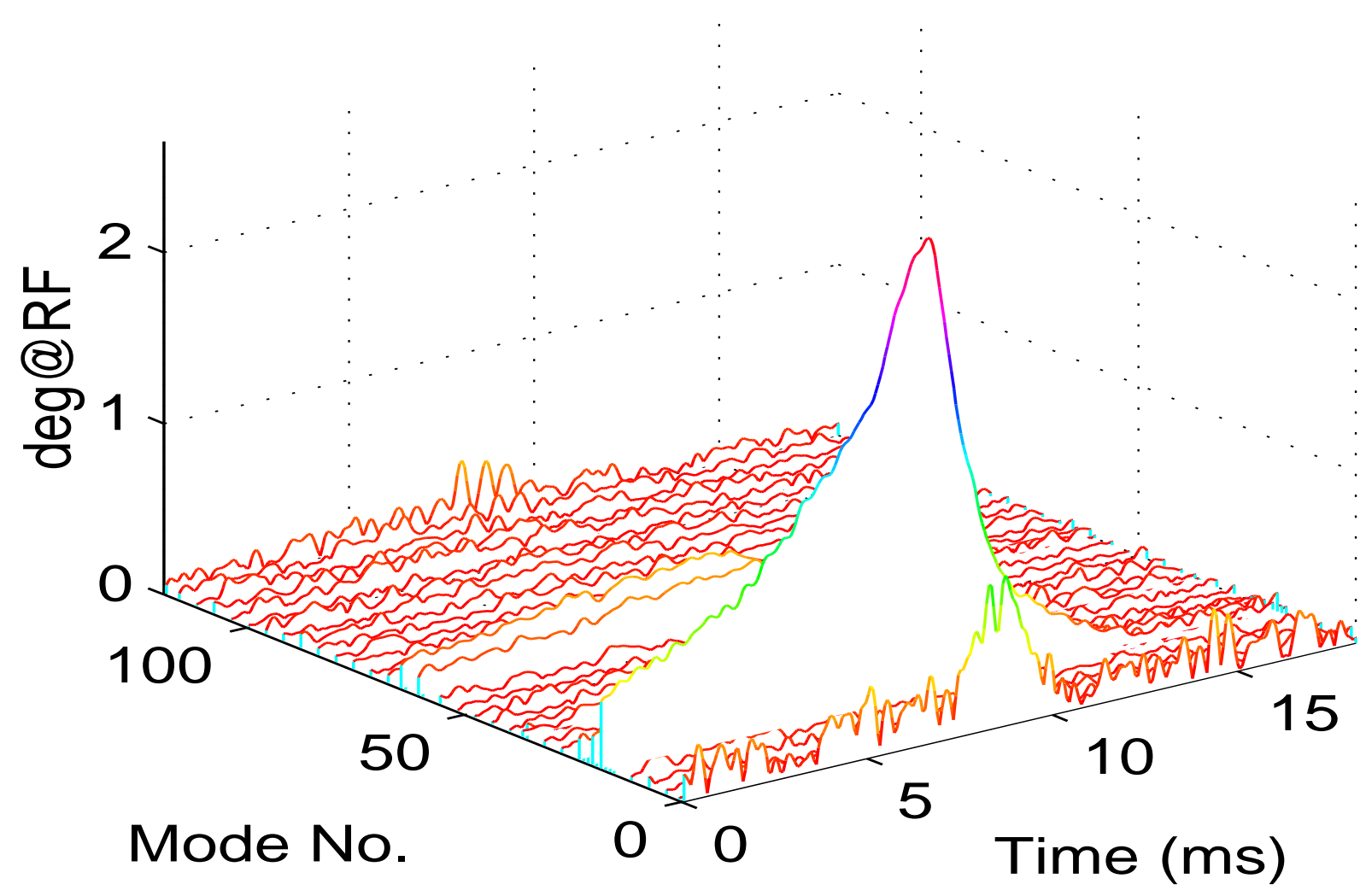

Figure 3: Modal longitudinal grow-damp transient from DA $\Phi$ NE. The feedback is turned off and mode 20 in the machine is unstable with a growth rate of $0.2 \mathrm{~ms}^{-1}$

\section{Prediction of Instability Thresholds}

As the growth rates of these instabilities are proportional to current, a threshold current exists for each mode for which the difference between a growth rate, and damping rate ( from radiation damping plus action of the feedback system) becomes positive. In an operational environment it is desirable to be able to predict the instability threshold ( for example, to confirm the configuration of the RF system) without having to dedicate a test fill to check a threshold directly. The grow-damp sequences, if measured above the no-feedback threshold, directly measure the gain margin at that specific current. If the measurements are repeated over a range of currents, a plot such as figure \#6 can be made. This figure shows the free growth rate of the PEP-II LER for the most unstable band of cavity HOMs vs. current. The linear scaling of growth rate with current is seen, and the projection of the line to the zero current position reveals the radiation damping in the system ( design value $\lambda_{\text {rad }}=0.03 \mathrm{~ms}^{-1}$ ). The threshold is clearly seen at $310 \mathrm{~mA}$ where the growth rate becomes positive. Related techniques, examining fed-back damping rates vs. current, and quantifying the degree of saturation in the feedback channel allow the prediction of a maximum current that can be 


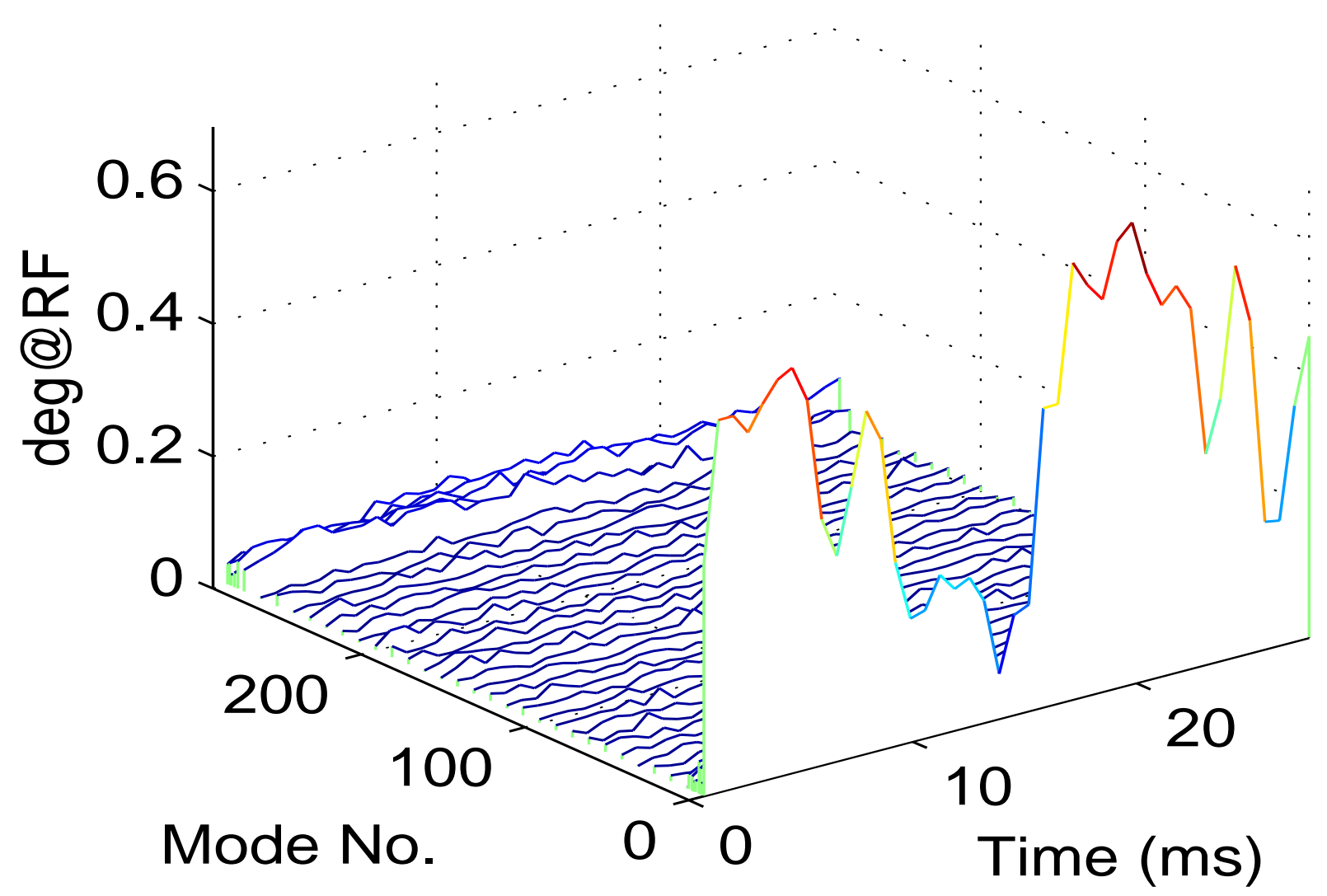

Figure 4: . Steady state feedback-on record of LER motion at $110 \mathrm{~mA}$, showing a disturbance which drives mode zero at roughly 0.3 degrees rms with significant time structure.

controlled with feedback from lower-current measurements. If the grow-damp sequences are perfomed with positive feedback, rather than no feedback for the exciting interval, these measurements can be made well below threshold and still predict the instability thresholds. Figure \#7 shows this sort of positive feedback excitation from SPEAR, revealing the two modes closest to instability. In a commissioning environment, in which operating currents may be below the design current, it has proven very useful to be able to predict high-current behavior, and operating margins, before exploring the high-current operating points directly.

\section{$5 \quad$ Transverse Bunch Train Studies}

During the PEP-II HER comissioning a detailed series of transverse instability measurements was made to try to understand the mechanism which was producing unexpectedly rapid growth rates in both vertical and horizontal planes [10]. Measurements were performed on a variety of fill patterns, including various even-fill patterns, and very asymmetric train patterns. The impedance spectrum sampled by these varied patterns is quite different, and 


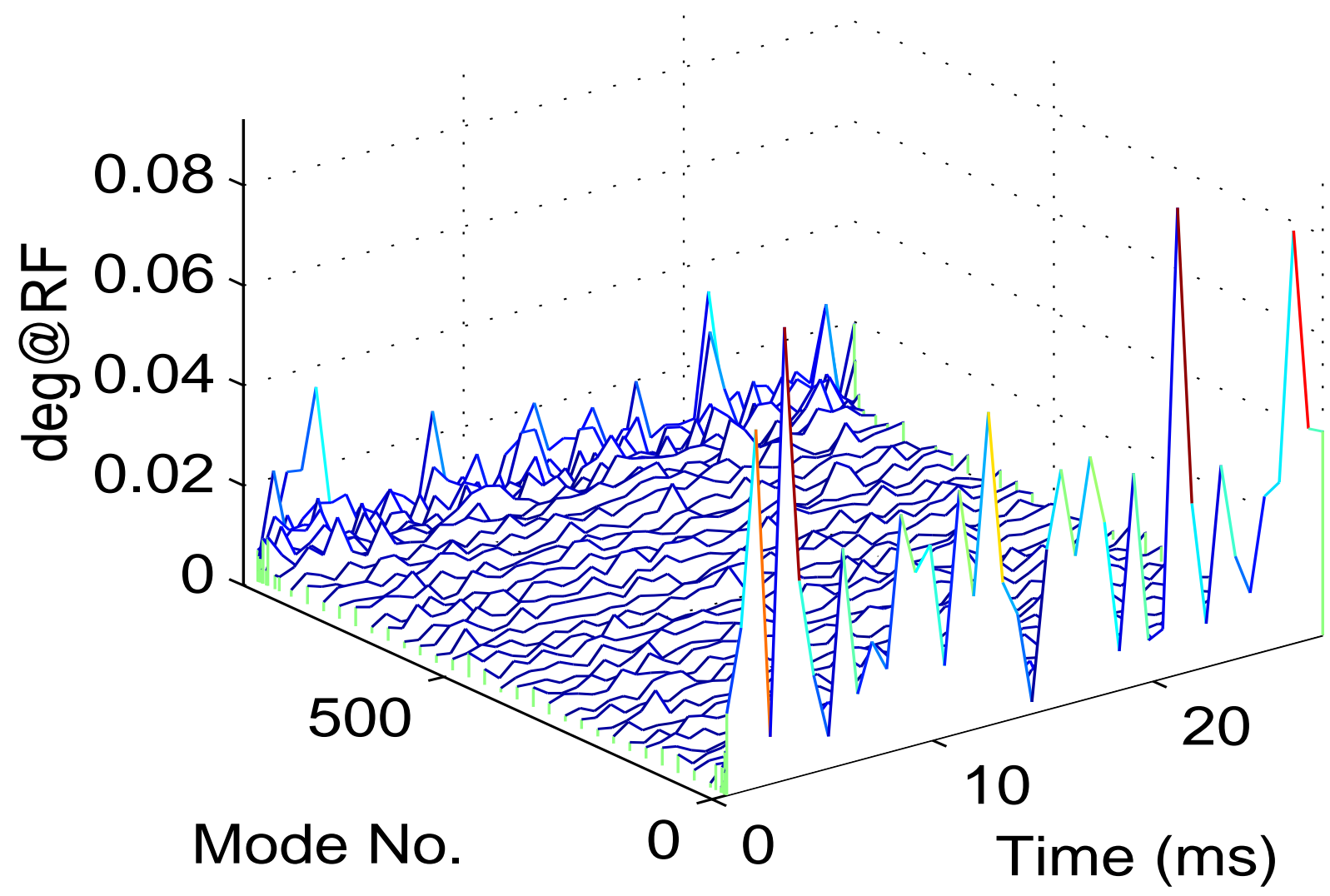

Figure 5: Steady state feedback-on record of LER motion (873 bucket pattern) at $730 \mathrm{~mA}$, after improvement of master oscillator phase noise. The mode zero motion is now roughly 0.03 degrees rms, while the remaining controlled bandwidth has residual noise of less than 0.005 degrees.

it is interesting to observe the evolution of instabilities in these bunch trains as a possible signature of ion instabilities [11]. Such a measurement from the PEP-II HER is shown in figure \#8, which shows the vertical rms amplitude of bunch motion along a 150 bunch train at 10 intervals during a $20 \mathrm{~ms}$ damp-grow sequence. Motion is clearly seen to originate from the tail of the train and propogate back towards the head, with an interesting secondary growing structure appearing around bucket 90 roughly $10 \mathrm{~ms}$ into the sequence. Also seen is a growth of the leading bunches during the transient in isolation from the motion at the tail.

Figure \#9 shows the average spectrum of each bunch during the transient. The motion is composed of a single eigenmode, and there is no measurable tune spread along the train. Such a tune spread would be strongly suggestive of a fast-ion type instability due to variations in the ion tune[12]. Figure \#10 is a presentation showing the angular position in phase space of each bunch vs. time for this $20 \mathrm{~ms}$ damp-grow transient. The reference phase is taken from bunch 150 ( the tail of the train). The figure shows several striking features. First, the anomalous amplitudes for the first 20 bunches are seen to be motion corresponding to a 180 


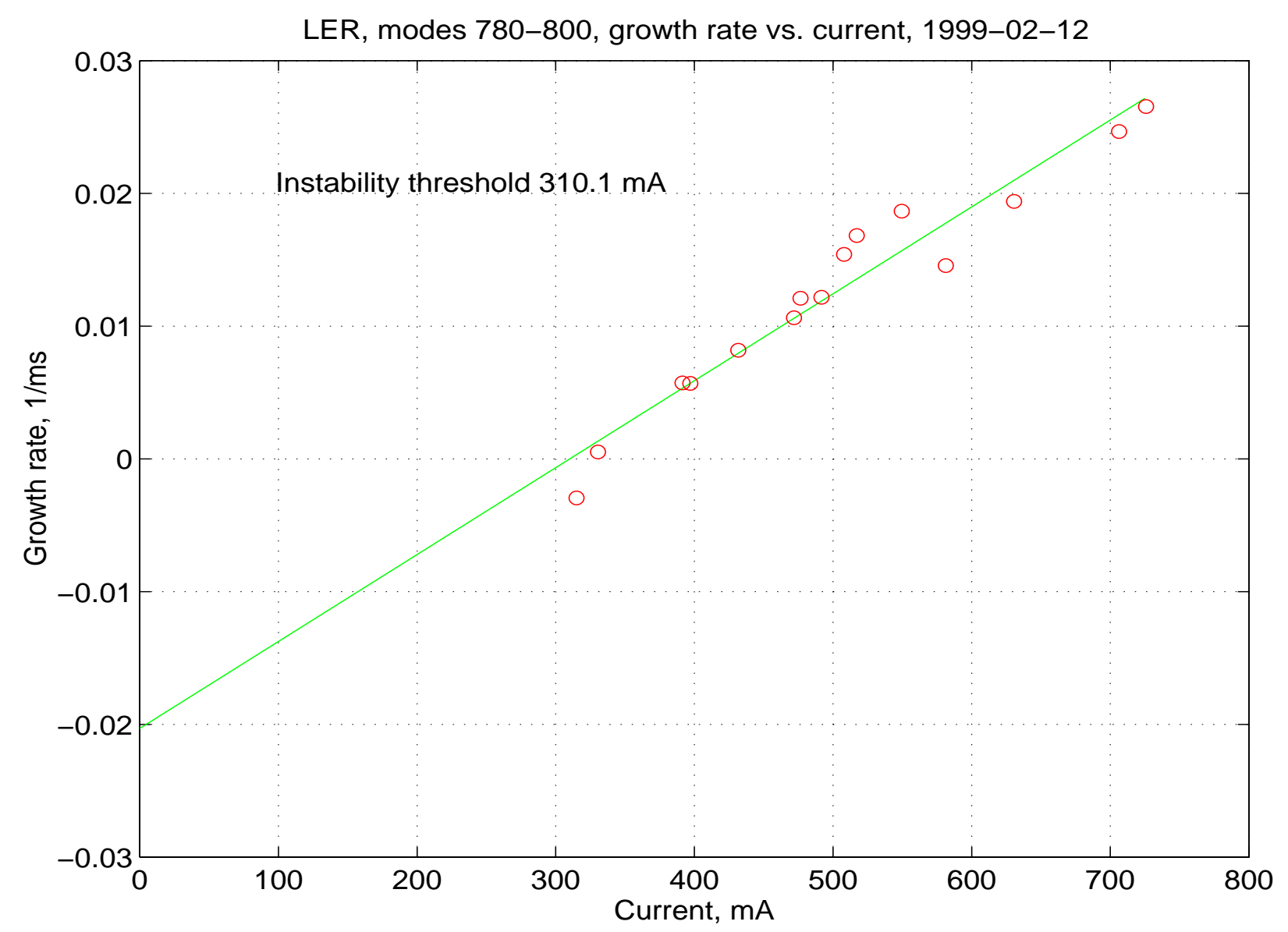

Figure 6: . PEP-II LER longitudinal growth rates vs. current for modes $780-800$

degree phase shift relative to the tail (other diagnostics indicate that these initial bunches are uncontrolled or driven by some mechanism, even in the damped state). The majority of the train of oscillators stays mostly fixed in phase space relationship during the growing transient. As the amplitude grows, the phase across the train uniformly shifts due to tune shifts with amplitude of motion. The unusual feature growing around bunch 90 after 10 $\mathrm{ms}$ is also seen as a phase perturbation in this figure, most likely from the same non-linear restoring mechanism which produces a tune shift with amplitude of motion.

A series of bunch train and uniform fill studies, in conjunction with deliberate variations of ring vacuum produced evidence which could be interpreted as suggestive of both ion-driven and HOM driven instabilities. The tail to head growing motion observed in the figure can be obtained from a resonator mechanism if the $\mathrm{Q}$ of the resonator is such that the energy stored in it is largely dissipated between turns in the ring. 


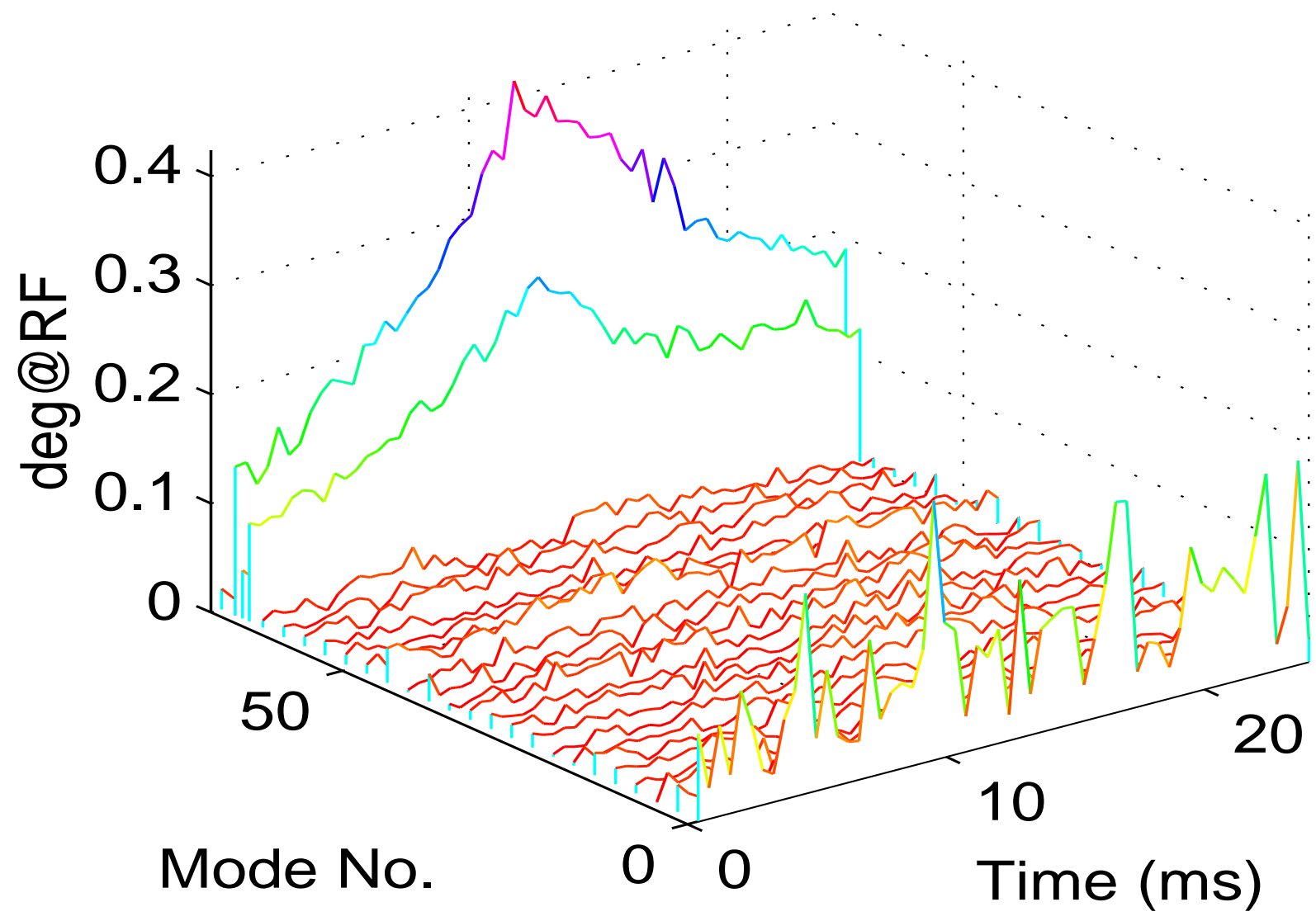

Figure 7: Grow-damp transient from SPEAR. The data is taken at $29 \mathrm{~mA}$, for which the machine is below threshold. Positive feedback is used to excite the growing portion of the transient, revealing modes 65 and 67 as the two modes closest to instability.

\section{$6 \quad$ Gap Transients and collider operation}

To help prevent ion instabilities the PEP-II design uses a current distribution with an unfilled gap of 5 to $15 \%$ of a revolution. Such a gap produces a significant transient in the RF cavity voltage, so that along the turn the individual bunches ride on unique synchronous phases. This effect produces a DC shift in the synchronous phase of the bunches vs. bunch number, such that the luminous region of collision moves if the LER and HER transients are not identical. The time-domain data from the motion recording directly shows this synchronous position of each bunch in the mean oscillation co-ordinate of each bunch. Figure \#10 shows the variation in synchronous phase for the PEP-II HER (at $319 \mathrm{~mA}$ ) and LER (at $638 \mathrm{~mA}$ ) machines in collision.

The variation in synchronous phase around the turn is associated with a variation in synchrotron frequency (tune) around the turn, which leads to a de-coupling of the bunches in the turn, and significantly raises the instability thresholds for such uneven fills $[13,14]$. 


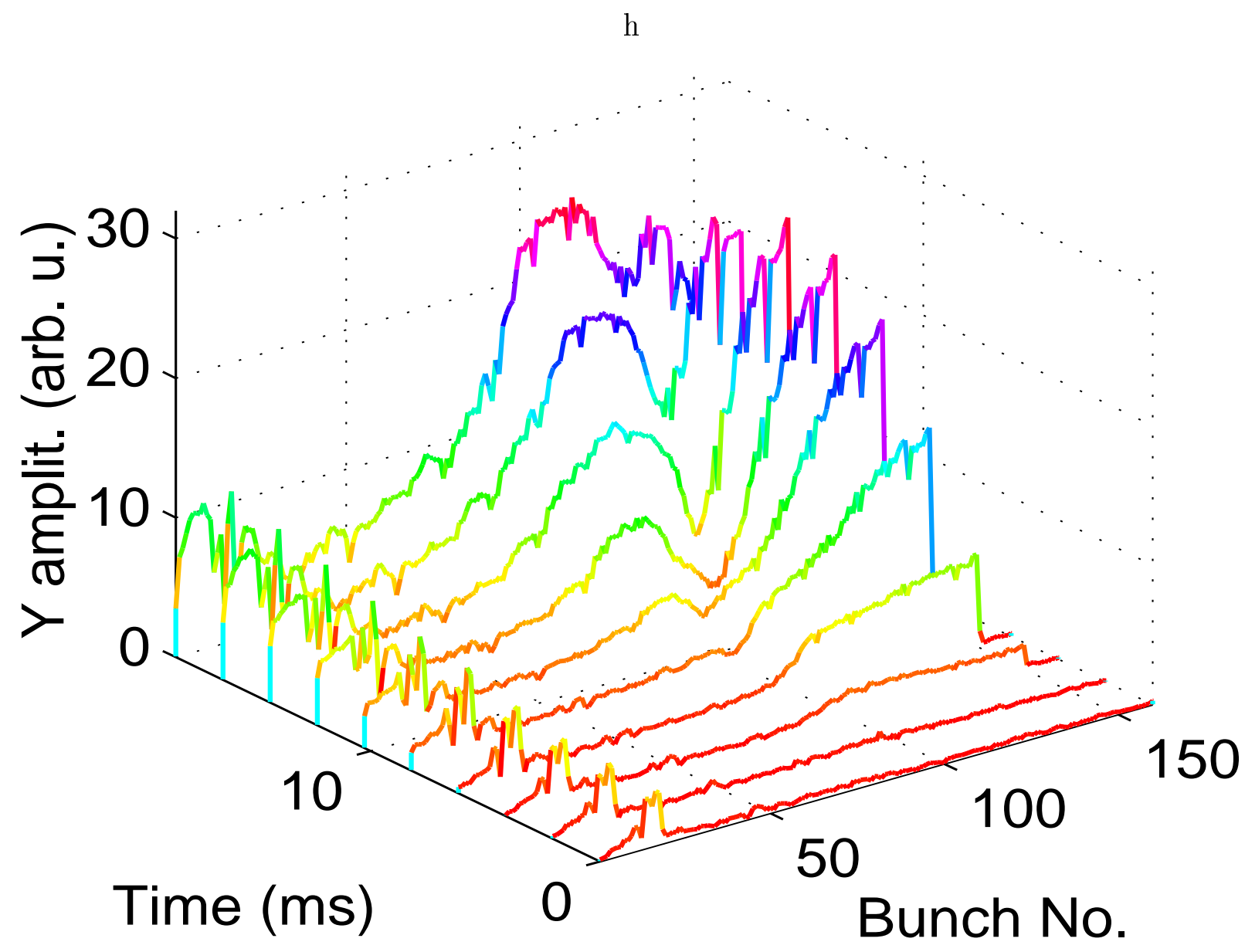

Figure 8: Vertical rms bunch motion for a 150 bucket train (150 buckets filled at 2 RF bucket spacing) for 10 successive instants during a $20 \mathrm{~ms}$ damp-grow measurement at $66 \mathrm{~mA}$. In this case feedback is turned off at $1.3 \mathrm{~ms}$. The first 20 bunches start out at a larger damped amplitude. The motion initially grows from the tail progressing towards the head of the train. 


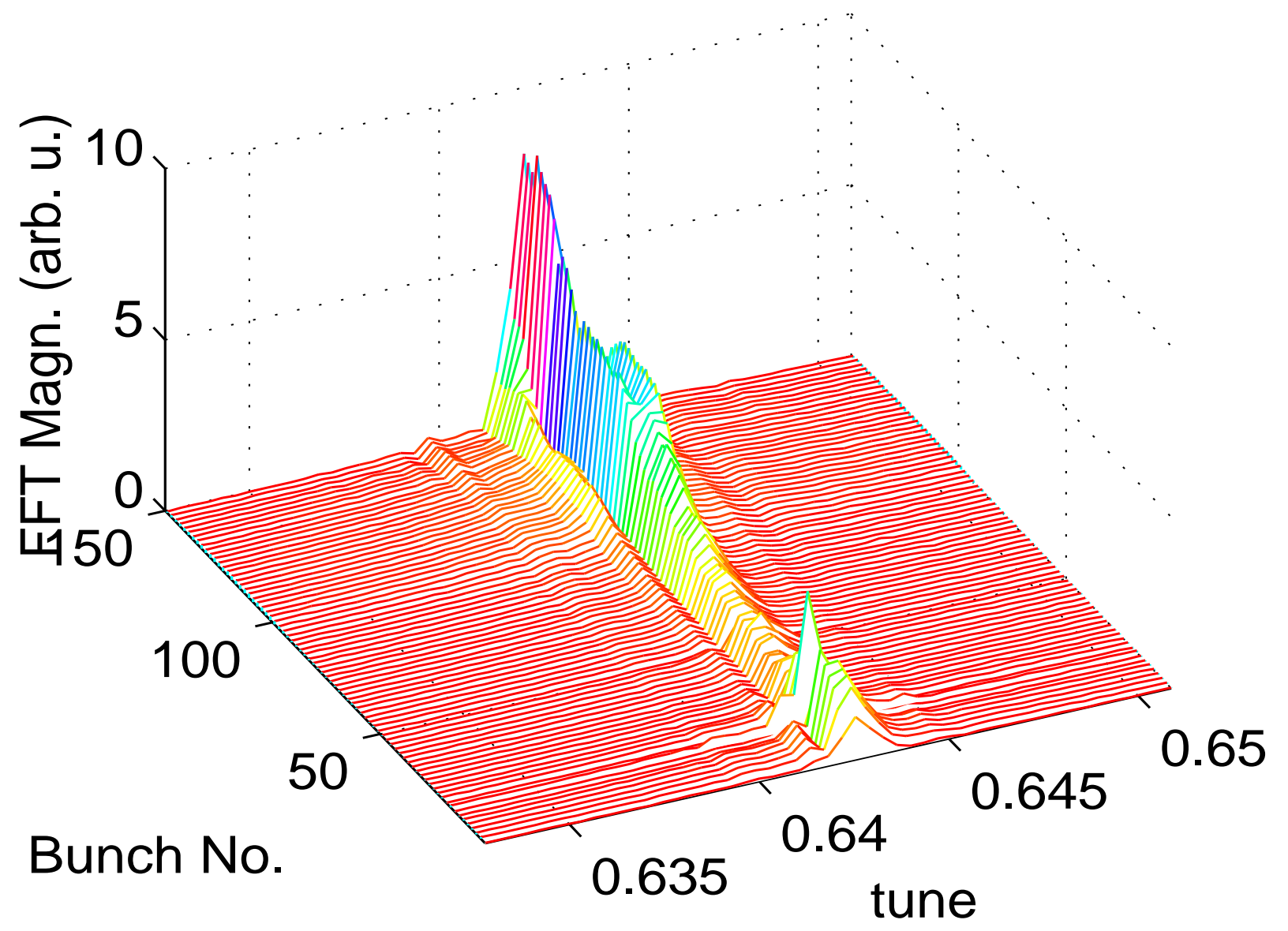

Figure 9: Average spectrum vs. bunch number for the 150 bucket train transient. The figure suggests only a single eigenmode is present in this data.

\section{$7 \quad$ Summary}

The original PEP-II/ALS/DA $\Phi$ NE longitudinal systems have been commissioned and we continue to develop longitudinal operating codes and longitudinal diagnostics. The programmable architecture and flexible structure of the longitudinal processing design has been extended to implement recording functions useful for transverse diagnostics. The information contained in the time-domain data, in conjunction with the development of new diagnostic codes, continues to suprise us with the variety and subtlety of the behavior of the beams.

\section{Acknowledgments}

The authors thank D. Andersen, W. Barry, J. Corlett, P. Corredoura, G. Lambertson, M. Minty, C. Limborg, W. Ross, J. Sebek, R. Tighe and U. Wienands for numerous thoughtful discussions and direct contributions of technical expertise. The operations groups at SPEAR, ALS, PEP-II and DA $\Phi$ NE have been essential during system commissioning. We also thank 
Boni Cordova-Grimaldi of SLAC for her patient fabrication of electronic components.

\section{References}

[1] A. Mosnier, "Cures of Coupled-Bunch Instabilities: HOM Free Cavities, Feedbacks, Laudau Damping" Proc. IEEE Particle Accelerator Conference, 1999

[2] M. Serio "Multi-Bunch Instabilities and Cures," Proc. European Particle Accelerator Conference, 1996.

[3] M. Tobiyama and E. Kikutani, "Commissioning of the KEKB Bunch Feedback Systems" Proc. IEEE Particle Accelerator Conference, 1999

[4] S. Guiducci, "DA $\Phi$ E Operating Experience," Proc. IEEE Particle Accelerator Conference, 1999.

[5] D. Teytelman, et al, "Beam Diagnostics Based on Time Domain Bunch by Bunch Data," Proc. Beam Instrumentation Workshop, 1998

[6] W. Barry, et al, " Operational Experience with the PEP-II Transverse Coupled-Bunch Feedback Systems", Proc. IEEE Particle Accelerator Conference, 1999

[7] S. Prabhakar et al, "Observation and Modal Analysis of Coupled-Bunch Longitudinal Instabilities via a Digital Feedback Control System," Particle Accelerators, 57/3, (1997).

[8] R. Tighe, "A Sampled Master Oscillator for the PEP-II B Factory," Proc. IEEE Particle Accelerator Conference, 1999.

[9] P. Corredoura, "Architecture and Performance of the PEP-II Low Level RF System," Proc. IEEE Particle Accelerator Conference, 1999.

[10] H.U. Wienands, et al, "Beam Commissioning of the PEP-II High Energy Ring," Proc. IEEE Particle Accelerator Conference, 1999

[11] J. Y. Huang, et al "Study of the Fast Beam-Ion Instability in the Pohang Light Source," Proc. European Particle Accelerator Conference, 1998.

[12] G. Stupakov, T. Raubenheimer, F. Zimmermann, "Fast Beam Ion Instability," Phys.Rev.E 52, p. 5499, 1995.

[13] S. Prabhakar, et al. "Commissioning Experience from PEP-II HER Longitudinal Feedback," Proc. Beam Instrumentation Workshop, 1998

[14] S. Prabhakar, et al. "Calculation of Impedance from Multibunch Synchronous Phases: Theory and Experimental Results," Proc. European Particle Accelerator Conference, 1998 


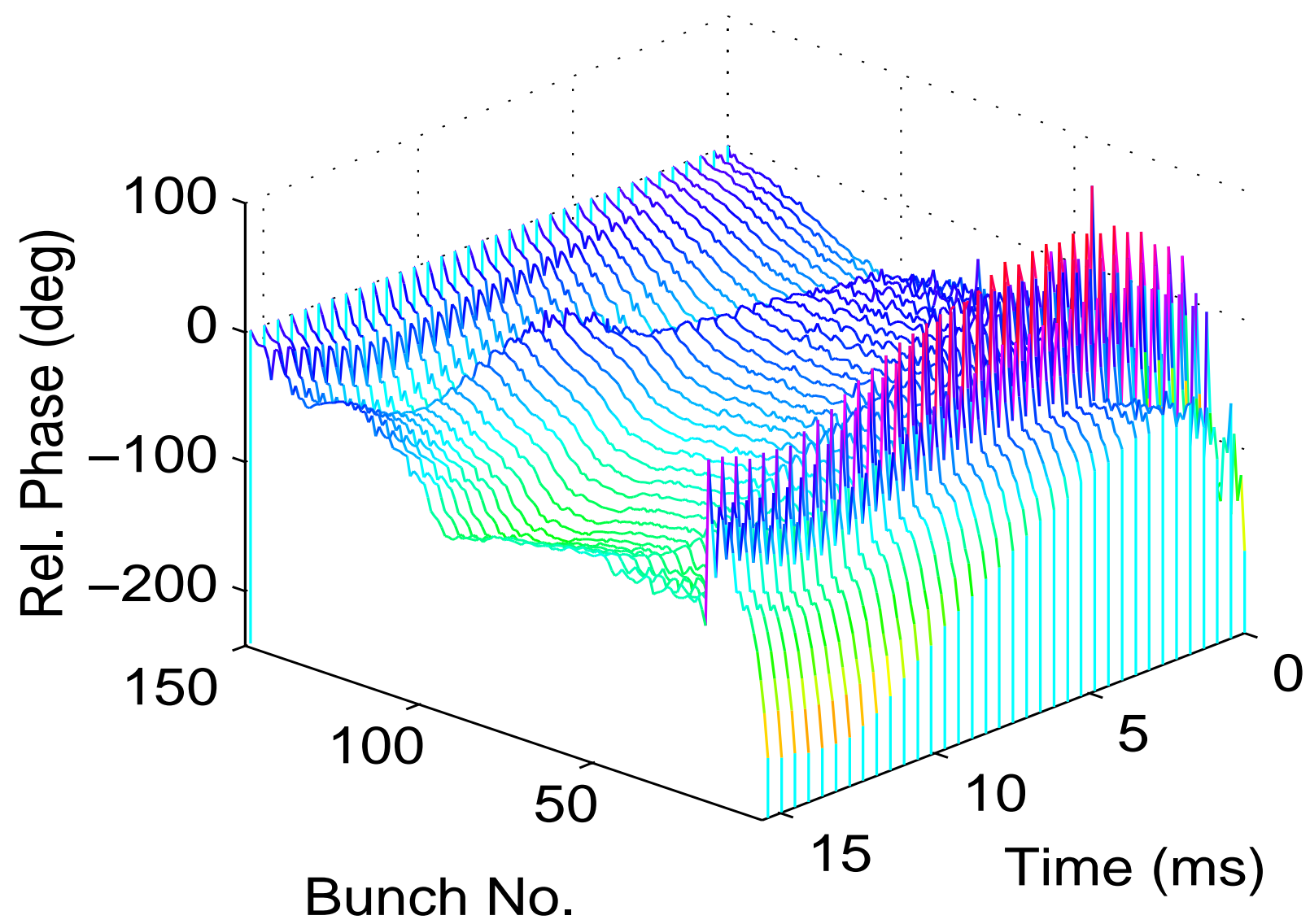

Figure 10: Surface plot showing the phase of each bunch vs. time for this $20 \mathrm{~ms}$ dampgrow transient. The reference phase is taken from bunch 150 ( the tail of the train). The figure shows several striking features. First, an anomalous 180 degree phase jump for the first 20 bunches. Second, as the bunch motion grows a general phase shift with amplitude is present, suggestive of some non-linear restoring potential which gives some tune dependence with amplitude. This non-linear amplitude effect is clearly seen in both the tail and around bunch 90 as the amplitudes of motion become large. 


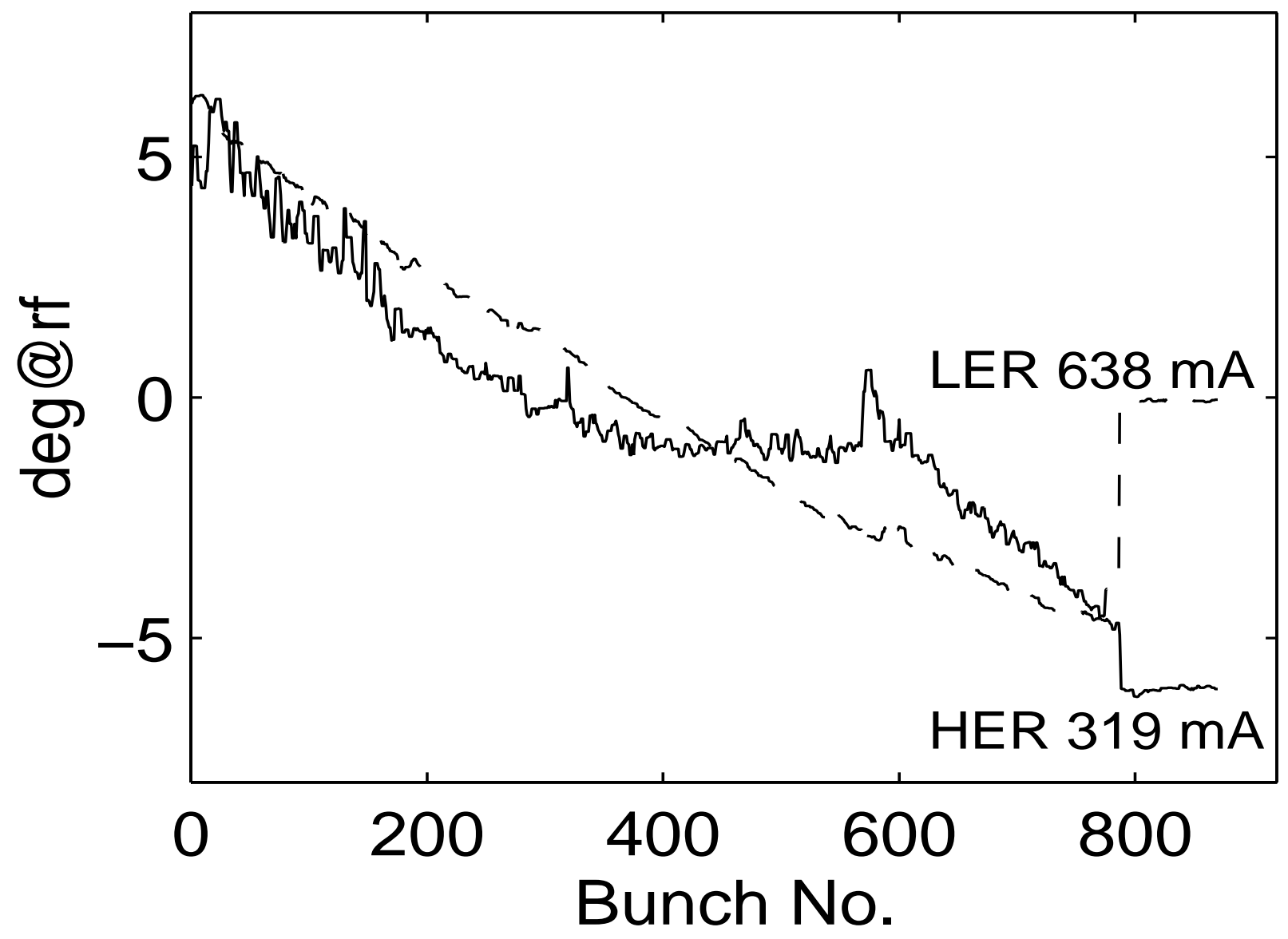

Figure 11: Synchronous phases of the LER ( $638 \mathrm{~mA})$ and HER ( $319 \mathrm{~mA})$ in collision ( 873 bucket pattern). 